\title{
Motivação do Aprendiz de Medicina: Uso da Escala de Motivação Acadêmica
}

\author{
Dejano T. Sobral \\ Universidade de Brasília
}

\begin{abstract}
RESUMO - O propósito do estudo foi analisar características da motivação de estudantes de medicina e sua relação com fatores acadêmicos no início do curso, pondo à prova uma versão da Escala de Motivação Acadêmica (EMA). O instrumento foi aplicado a 269 sujeitos de ambos os sexos, sendo reaplicada em $25 \%$ após um ano. Foram obtidas medidas subjetivas e objetivas do aprendizado, representando fatores motivacionais. Procedimentos estatísticos foram efetuados para configurar a validade interna e externa, incluindo análises de correlação entre componentes da EMA e as medidas seletivas. Os achados revelaram níveis satisfatórios de consistência interna, estabilidade temporal moderada e matriz de correlação das subescalas geralmente consistente. O perfil de respostas mostrou predominância de motivação autônoma e variação sexual. As correlações entre componentes da EMA e antecedentes e conseqüências motivacionais revelaram espectro de motivação compatível com influências individuais e contextuais. Os resultados dão suporte à validade do instrumento e ao seu uso no estudo de motivação universitária.
\end{abstract}

Palavras-chave: motivação; estudantes de medicina; aprendizagem e desempenho; Escala de Motivação Acadêmica.

\section{Learner's Motivation in Medical Studies: Use of the Academic Motivation Scale}

\begin{abstract}
ABSTARCT - The purpose of this work was to analyze features of students' motivation in relation to academic factors after one year of medical studies, testing out a version of the Academic Motivation Scale (AMS). The AMS was administered to 299 subjects of both sexes and re-administered to a $25 \%$ sample one year later. Measures of learning perceptions and achievement representing motivational factors were also obtained. Statistical procedures were done to describe the internal and external validity, including correlation analysis between the AMS components and the motivational measures. The findings showed satisfactory levels of internal consistency, moderate temporal stability and consistent inter-correlation matrix. The profile of responses revealed a predominance of autonomous motivation and a gender effect. The relationships of the AMS components with the motivational antecedents and consequences revealed a spectrum of motivation compatible with individual and contextual influences. The results support the validity of the AMS and its usefulness in the study of academic motivation.
\end{abstract}

Key words: motivation; medical students; learning and performance; Academic Motivation Scale.

No olhar acadêmico, motivação é um conceito que abarca diversas conotações ou construtos significativamente relacionados à aprendizagem e ao desenvolvimento educativo e que tem gerado múltiplas perspectivas de estudo. Pintrich (1991) identificou três categorias gerais de construtos pertinentes à motivação no contexto educativo: (a) crenças dos indivíduos em suas capacidades para realizar uma atividade; (b) suas razões ou propósitos para se engajarem na atividade e (c) suas reações afetivas em relação à atividade. Numa revisão recente, Murphy e Alexander (2000) examinaram 20 termos fundamentais vinculados às diferentes linhas ou perspectivas de investigação nesse campo.

A teoria da autodeterminação orienta uma dessas importantes linhas de investigação e postula que contextos que dão suporte a necessidades psicológicas básicas (autonomia,

1 O autor registra o apoio institucional, agradece a participação ativa e o interesse real dos aprendizes envolvidos e expressa seu reconhecimento ao Dr. Robert J. Vallerand por fornecer o instrumento original e permitir a realização do estudo.

2 Endereçco: Faculdade de Medicina - C.P. 04569 - 70919-970 Brasília -DF.E-mail: dtsobral@unb.br/dtsobral@netscape.net competência e conectividade) promovem ação intencional ou motivada (Decci \& Ryan, 1985). Na perspectiva da teoria da autodeterminação, os indivíduos diferem em relação ao nível e ao tipo de motivação. A primeira característica traduz a intensidade enquanto a segunda se refere ao porquê da motivação, isto é, as atitudes e os propósitos subjacentes. Dois tipos básicos de motivação são caracterizados: motivação intrínseca, em que se faz algo pelo interesse e prazer inerentes à ação; e motivação extrínseca, em que se faz algo por causa de conseqüência ou desfecho distinto da ação. A motivação extrínseca assume diferentes formas, que se distinguem pelo grau de internalização e integração de valores e regulação de condutas, refletindo diferentes graus de autonomia. Uma motivação autônoma relaciona-se com mais qualidade no aprendizado, maior persistência e melhor ajuste psicológico dos aprendizes, do ensino primário ao ensino superior (Decci, Vallerand, Pelletier \& Ryan, 1991; Deci, Ryan \& Williams, 1996; Ryan \& Decci, 2000).

Os princípios da teoria de autodeterminação são pertinentes para a educação profissional, na medida em que a diferenciação e expressão dos tipos primários de motivação têm implicações para os múltiplos desfechos da aprendiza- 
gem. O contexto da formação médica, por exemplo, pode favorecer distintamente a expressão de motivação autônoma (primariamente intrínseca) ou de motivação controlada (extrínseca) nos aprendizes, com efeitos na própria postura profissional (Williams, Saizow \& Ryan, 1999).

Em que medida a experiência inicial do curso se reflete no espectro de motivação de estudantes de medicina? Sabe-se que ao ingressar no curso, a grande maioria dos alunos de medicina têm perspectivas quanto à prática médica e não quanto à atividade acadêmica em si. Isso se revela pelos fatores de influência na escolha da carreira, em que sobressaem a necessidade de contacto com pacientes como pessoas, o interesse em ajudar o próximo e a busca de independência (Sobral, 1977). Tradicionalmente, contudo, o primeiro ano de estudos é dedicado ao estudo de ciências básicas, especialmente no campo biológico, o que poderia levar a um conflito de orientação ou mesmo desmotivação do estudante. Na concepção desenvolvida por Ryan \& Deci (2000), o ambiente acadêmico pode facilitar ou inibir a motivação intrínseca (para atividades que atraiam o interesse intrínseco) em decorrência do suporte ou obstrução das necessidades psicológicas de competência e autonomia dos aprendizes. Percepções objetivas ou subjetivas sobre o nível de eficácia pessoal ou competência nesse ambiente de estudos acadêmicos poderiam afetar a intensidade ou a orientação da motivação.

O propósito geral do estudo empreendido foi analisar o nível e a orientação de motivação de estudantes de medicina e sua relação com fatores acadêmicos, na fase inicial do curso de graduação, sob a perspectiva da teoria da autodeterminação. Utilizou-se uma versão da Escala de Motivação Acadêmica, que se baseia nos princípios dessa teoria (Vallerand, Blais, Brière \& Pelletier, 1989). Esse instrumento tem mostrado propriedades psicométricas robustas em vários estudos, em termos de consistência interna, estabilidade temporal e validade de construto, conforme Vallerand e cols. (1992; 1993).

O presente trabalho tem os seguintes objetivos:

1. Conferir propriedades da versão da Escala de Motivação Acadêmica (EMA).

2. Determinar o perfil de respostas à EMA, com relação ao gênero dos estudantes.

3. Analisar as relações dessas respostas com a autoconfiança na aprendizagem, as percepções sobre o valor e o significado do aprendizado, o rendimento acadêmico e a intenção dos estudantes de prosseguir os estudos.

\section{Método}

\section{Sujeitos}

A população alvo compreendia estudantes de medicina da Universidade de Brasília, que seguiram o acompanhamento curricular implantado a partir de 1988. O critério de inclusão, por amostragem consecutiva, foi a passagem do aluno para o terceiro semestre do curso no decurso de quatro anos, entre 1998 e 2001. A amostra de 269 sujeitos (55,8\% masculinos) correspondia a $85,7 \%$ do total de alunos nessa posição do fluxo do curso, durante o período do estudo. A média de idade dos sujeitos era 20,5 anos, na época de participação.

\section{Medidas}

Escala de Motivação Acadêmica. Esta escala é constituída de 28 itens subdivididos em sete subescalas: três correspondem a tipos de motivação intrínseca; outras três incorporam tipos de motivação extrínseca e, a última, representa desmotivação ou ausência de motivação. Os tipos de motivação intrínseca abrangem: (a) motivação intrínseca para saber (fazer algo pelo prazer e satisfação que decorre de aprender, explorar ou entender); (b) motivação intrínseca para realizar coisas (fazer algo pelo prazer e satisfação que decorre da busca de realização ou criação de coisas); e (c) motivação intrínseca para vivenciar estímulo (fazer algo a fim de experimentar sensações estimulantes, de natureza sensorial ou estética). Os tipos de motivação extrínseca incluem: (d) regulação por identificação (fazer algo porque se decidiu fazê-lo); (e) regulação por introjeção (fazer algo porque se pressiona a si próprio a fazê-lo); (f) regulação externa (fazer algo porque se sente pressionado por outros a fazê-lo). Por fim, o conceito de desmotivação (amotivation) implica ausência de percepção de contingências entre as ações e seus desfechos (falta de motivos intrínsecos ou extrínsecos).

$\mathrm{O}$ formato para ensino superior do instrumento (Vallerand e cols., 1992; 1993) foi traduzido para o Português, usandose procedimentos apropriados para tradução transcultural. A versão utilizada no estudo lista 28 itens que podem representar razões para o estudante vir à universidade. Para efeito deste estudo, acrescentou-se uma escala analógica visual indagando o grau de motivação do aprendiz para o prosseguimento dos estudos em medicina. $\mathrm{O}$ instrumento completo consta do anexo.

Inventário de Valorização do Curso. O Inventário de Valorização do Aprendizado (Nehari \& Bender, 1978) é constituído de 36 itens que abrangem aspectos dos desfechos de aprendizado de conteúdo e de desenvolvimento pessoal na experiência educativa. As respostas ao inventário expressam as percepções sobre o valor e o significado do aprendizado e relacionam-se significativamente com as reações afetivas dos aprendizes (Sobral, 1992). O instrumento foi aplicado com referência à experiência curricular nos dois primeiros semestres do fluxograma do curso. Na amostra do estudo, a consistência interna revelou-se muito boa (alfa de Cronbach $=0,94$ ).

Escala de autoconfiança. A autoconfiança como aprendiz foi medida por escala analógica visual (Sobral, 1993). A escala tem índice de estabilidade temporal satisfatória e serve de indicador de auto-eficácia, no sentido definido por Bandura (1989).

Rendimento acadêmico. Foram obtidos indicadores de rendimento antes e depois da aplicação da ERA: (1) rendimento acadêmico no primeiro ano (média ponderada cumulativa correspondente às disciplinas dos dois primeiros semestres); e (2) rendimento acadêmico no terceiro semestre (média ponderada das disciplinas do terceiro semestre).

\section{Contexto e Procedimentos}

A coleta de dados dos inventários foi realizada no início do terceiro semestre do curso, no ambiente da aprendizagem pré-clínica. Uma amostra de 70 participantes completou a 
Escala de Motivação Acadêmica uma segunda vez, já no ambiente de aprendizagem clínica, 12 meses, em média, após a primeira administração.

\section{Análise dos Dados}

Vários procedimentos estatísticos principais foram utilizados para caracterizar a consistência interna e estabilidade da EMA, o perfil de respostas dos sujeitos e as inter-relações com as medidas motivacionais seletivas: (a) análise de confiabilidade; (b) coeficientes de correlação para medir a associação entre pares de variáveis; (c) testes t, ou análises de variância, para aferir diferenças entre médias de grupos; (d) análise de regressão múltipla para verificar a associação entre fatores explanatórios e a variável de desfecho, quando outros fatores são controlados.

Os trabalhos de Andrew, Klem, Davidson, O' Malley e Rodgers (1981), Norman e Streiner (1994) e Streiner e Norman (1995), orientaram os procedimentos de análise. As operações estatísticas foram realizadas no pacote SPSS, versão 9.1 .

\section{Resultados}

O valor médio dos índices (alfa) de consistência interna das subescalas foi 0,78 - variando entre 0,66 (motivação intrínseca para vivenciar estímulos) e 0,85 (motivação intrínseca para saber). A Tabela 1 mostra esses índices e a matriz de intercorrelação das sete subescalas ordenadas segundo um continuum de autodeterminação. As correlações entre as subescalas acompanham o denominado modelo simplex descrito por Vallerand e cols. (1993), com algumas restrições. Os componentes de motivação intrínseca mostraram correlações elevadas e positivas entre si, bem como correlações negativas com a subescala de desmotivação. Observou-se, porém, forte associação entre as subescalas de introjeção e de motivação intrínseca para realização; tal associação indica interações distintivas dos sujeitos e seu contexto acadêmico.

A estabilidade temporal das diversas subescalas mostrou variação expressiva, em torno do valor médio $(r=0,51)$ : foi menor para o componente de desmotivação $(r=0,43)$ e maior para a motivação intrínseca para saber $(r=0,59)$. Para comparação, a correlação emparelhada das duas medidas de motivação para prosseguir os estudos foi claramente maior $(\mathrm{r}=0,68)$. Os níveis de estabilidade são moderados, mas parecem aceitáveis considerando-se que as medidas foram tomadas em contextos diversos e distantes cerca de 12 meses entre si. Observe-se que houve diferença significante entre as médias das medidas temporais no caso de duas subescalas, que refletem níveis distintos de autodeterminação: identificação, em que houve elevação, e desmotivação, em que houve diminuição, da primeira para a segunda medida.

$\mathrm{Na}$ análise de variância (repeated measures) as médias das sete subescalas diferiam significantemente entre si, exceto na comparação entre motivação para realização e motivação para vivenciar estímulos. Os componentes mais significativos foram, em ordem decrescente: identificação, motivação intrínseca para saber, motivação intrínseca para realização e motivação intrínseca para vivenciar estímulos. Observou-se ainda interação significante entre subescalas e gênero $(F=$

Tabela 1. Coeficientes de intercorrelação das subescalas da Escala de Motivação Acadêmica (EMA) em amostra de estudantes de medicina (N=299)

\begin{tabular}{|c|c|c|c|c|c|c|c|}
\hline Subescalas da EMA & 1 & 2 & 3 & 4 & 5 & 6 & 7 \\
\hline 1. Motivação intrínseca para saber & 0,85 & 0,52 & 0,57 & 0,47 & 0,19 & 0,11 & $-0,39$ \\
\hline 2. Motivação intrínseca para realização & & 0,81 & 0,52 & 0,29 & 0,59 & 0,20 & $-0,20$ \\
\hline 3. Motivação intrínseca para vivenciar estímulo & & & 0,66 & 0,44 & 0,29 & 0,12 & $-0,39$ \\
\hline 4. Motivação extrínseca - identificação & & & & 0,75 & 0,17 & 0,27 & $-0,40$ \\
\hline 5. Motivação extrínseca - introjeção & & & & & 0,83 & 0,46 & 0,07 \\
\hline 6. Motivação extrínseca - controle externo & & & & & & 0,82 & 0,09 \\
\hline 7. Desmotivação & & & & & & & 0,71 \\
\hline
\end{tabular}

Valores na diagonal representam o índice de confiabilidade (Cronbach alfa) da subescala.

Tabela 2. Correlação e diferença entre medidas emparelhadas das subescalas da Escala de Motivação Acadêmica (EMA), com intervalo médio de 12 meses, em subamostra de estudantes de medicina $(N=70)$

\begin{tabular}{|c|c|c|c|c|c|}
\hline Subescalas da EMA & $\begin{array}{l}1^{\text {a }} \text { Medida } \\
\text { Médias (dp) }\end{array}$ & $\begin{array}{c}2^{\mathrm{a}} \text { Medida } \\
\text { Médias (dp) }\end{array}$ & $\begin{array}{c}\text { Correlação } \\
\mathbf{r}\end{array}$ & Diferença & $\begin{array}{c}\text { Teste } t \\
\mathbf{p}\end{array}$ \\
\hline 1. Motivação intrínseca para saber & $23,51 \quad(3,09)$ & $23,70 \quad(3,47)$ & 0,59 & 0,17 & 0,52 \\
\hline 2. Motivação intrínseca para realização & $19,40 \quad(4,98)$ & $19,80 \quad(4,39)$ & 0,45 & 0,40 & 0,50 \\
\hline 3. Motivação intrínseca para vivenciar estímulo & $18,79(4,20)$ & $19,17 \quad(5,09)$ & 0,50 & 0,39 & 0,49 \\
\hline 4. Motivação extrínseca - identificação & $24,90 \quad(3,11)$ & $25,47 \quad(2,98)$ & 0,57 & 0,57 & 0,09 \\
\hline 5. Motivação extrínseca - introjeção & $13,94(5,60)$ & $14,94(6,24)$ & 0,49 & 1,00 & 0,17 \\
\hline 6. Motivação extrínseca - controle externo & $18,64(5,22)$ & $18,97 \quad(5,38)$ & 0,56 & 0,33 & 0,58 \\
\hline 7. Desmotivação & $6,06 \quad(2,94)$ & $5,37 \quad(2,02)$ & 0,43 & $-0,69$ & 0,04 \\
\hline
\end{tabular}


$3,5 ; \mathrm{p}<0,01)$. Estudantes de sexo feminino mostraram escores significantemente superiores na motivação intrínseca para realização, enquanto os de sexo masculino revelaram escores significantemente superiores na motivação extrínseca sob controle externo. As médias dos componentes da EMA, no conjunto e por gênero dos estudantes, são apresentadas na Tabela 3.

Foram medidas as correlações entre os componentes da EMA e três índices que representam antecedentes motivacionais: o rendimento acadêmico, o escore do IVC e o nível de autoconfiança como aprendiz, ao término do primeiro ano de estudos. O IVC revela as percepções entretidas pelos estudantes quanto ao valor e o significado do aprendizado prévio: o escore total mostrou correlações positivas e significantes com as subescalas de motivação intrínseca e a subescala de identificação, bem como correlação negativa com a subescala de desmotivação. O padrão de correlação do índice de rendimento acadêmico com as subescalas foi semelhante, mas em nível mais fraco em todos os casos. O nível de autoconfiança como aprendiz apresentou, também, correlações positivas e significantes com os três componentes de motivação intrínseca, bem como correlação negativa com a subescala de desmotivação. A Tabela 4 mostra os dados quantitativos, que são geralmente consistentes com as expectativas.

Foram medidas, também, as correlações entre os componentes da EMA e duas variáveis que correspondem a conseqüências motivacionais: o rendimento acadêmico no terceiro semestre e o grau de motivação para prosseguir os estudos. Essa última mostrou correlações positivas e significantes com as três subescalas de motivação intrínseca e a subescala de identificação, bem como correlação negativa significante com a subescala de desmotivação. As correlações do rendimento acadêmico, no primeiro ano e no terceiro semestre, com as sete subescalas foram quase idênticas, indicando estabilidade no espectro de motivação em relação a esse fator motivacional.

Uma análise de regressão múltipla foi realizada procurando aferir a contribuição relativa dos componentes da EMA

Tabela 3. Médias e desvios padrões (dp) de subescalas da Escala de Motivação Acadêmica em estudantes de medicina em conjunto e agrupados por gênero

\begin{tabular}{|c|c|c|c|c|}
\hline Subescalas da EMA & $\begin{array}{c}\text { Total }(\mathrm{N}=299) \\
\text { Médias (dp) }\end{array}$ & $\begin{array}{l}\text { M (n = 150) } \\
\text { Médias (dp) }\end{array}$ & $\begin{array}{c}\text { F }(\mathbf{n}=119) \\
\text { Médias (dp) }\end{array}$ & $\begin{array}{c}\text { Teste } t \\
\mathbf{p}\end{array}$ \\
\hline 1. Motivação intrínseca para saber & $23,75 \quad(3,49)$ & $23,42 \quad(3,45)$ & $24,16(3,52)$ & 0,084 \\
\hline 2. Motivação intrínseca para realização & $19,75 \quad(5,02)$ & $19,20 \quad(5,09)$ & $20,45 \quad(4,88)$ & 0,043 \\
\hline 3. Motivação intrínseca para vivenciar estímulo & $19,20 \quad(4,35)$ & $18,94(4,50)$ & $19,54(4,15)$ & 0,264 \\
\hline 4. Motivação extrínseca - identificação & $24,78 \quad(3,35)$ & $24,49 \quad(3,54)$ & $25,15 \quad(3,06)$ & 0,109 \\
\hline 5. Motivação extrínseca - introjeção & $13,92 \quad(5,99)$ & $13,53(6,05)$ & $14,42(5,89)$ & 0,225 \\
\hline 6. Motivação extrínseca - controle externo & $17,92 \quad(5,69)$ & $18,68(5,76)$ & $16,96(5,46)$ & 0,013 \\
\hline 7. Desmotivação & $5,82(2,76)$ & $6,09 \quad(2,83)$ & $5,48 \quad(2,63)$ & 0,069 \\
\hline
\end{tabular}

Tabela 4. Correlação de subescalas da Escala de Motivação Acadêmica (EMA) com três antecedentes motivadores: rendimento acadêmico no primeiro ano, escore do inventário de valorização do curso (IVC) e nível de autoconfiança como aprendiz (N = 269)

\begin{tabular}{lccc}
\hline \multicolumn{1}{c}{ Subescalas da EMA } & $\begin{array}{c}\text { Rendimento } \\
\text { acadêmico }\end{array}$ & Valorização do curso & $\begin{array}{c}\text { Autoconfiança como } \\
\text { aprendiz }\end{array}$ \\
\hline 1. Motivação intrínseca para saber & 0,20 & 0,45 & 0,12 \\
2. Motivação intrínseca para realização & 0,11 & 0,43 & 0,18 \\
3. Motivação intrínseca para vivenciar estímulo & 0,20 & 0,41 & 0,14 \\
4. Motivação extrínseca - identificação & 0,13 & 0,22 & 0,06 \\
5. Motivação extrínseca - introjeção & 0,01 & 0,12 & 0,08 \\
6. Motivação extrínseca - controle externo & $-0,03$ & $-0,03$ & 0,07 \\
7. Desmotivação & $-0,13$ & $-0,36$ & $-0,16$ \\
\hline
\end{tabular}

Nota: $\boldsymbol{r}>0,11 \boldsymbol{p}<0,05 ; \boldsymbol{r}>0,15 \boldsymbol{p}<0,01 ; \boldsymbol{r}>0,21 \boldsymbol{p}<0,001$

Tabela 5. Correlação de subescalas da Escala de Motivação Acadêmica (EMA) com dois desfechos de motivação: rendimento acadêmico no terceiro semestre e grau de motivação para prosseguir os estudos $(\mathrm{N}=269)$

\begin{tabular}{lcc}
\hline \multicolumn{1}{c}{ Subescalas da EMA } & Rendimento acadêmico & Motivação para prosseguir \\
\hline 1. Motivação intrínseca para saber & 0,18 & 0,42 \\
2. Motivação intrínseca para realização & 0,09 & 0,33 \\
3. Motivação intrínseca para vivenciar estímulo & 0,18 & 0,42 \\
4. Motivação extrínseca - identificação & 0,14 & 0,31 \\
5. Motivação extrínseca - introjeção & 0,02 & 0,01 \\
6. Motivação extrínseca - controle externo & 0,04 & $-0,06$ \\
7. Desmotivação & $-0,10$ & $-0,58$ \\
\hline
\end{tabular}

Nota: $r>0,11 p<0,05 ; r>0,15 p<0,01 ; r>0,21 p<0,001$ 
Tabela 6. Predição do grau de motivação para prosseguir os estudos por componentes da EMA e medidas antecedentes (análise de regressão múltipla, stepwise)

\begin{tabular}{|c|c|c|c|c|c|}
\hline \multirow{2}{*}{ Medidas (índices/escores) } & \multirow{2}{*}{ beta } & \multirow{2}{*}{$\mathbf{t}$} & \multirow{2}{*}{ significância } & \multicolumn{2}{|c|}{ Correlação } \\
\hline & & & & total & parcial \\
\hline (Constante) & & 6,85 & 0,000 & & \\
\hline Desmotivação & 0,427 & $-8,51$ & 0,000 & $-0,583$ & $-0,463$ \\
\hline Autoconfiança como aprendiz & 0,222 & 4,77 & 0,000 & 0,354 & 0,282 \\
\hline Valorização do aprendizado & 0,167 & 3,24 & 0,001 & 0,442 & 0,195 \\
\hline Motivação para estímulos & 0,158 & 3,09 & 0,002 & 0,423 & 0,187 \\
\hline
\end{tabular}

Sumário do modelo de regressão: $\mathbf{R}^{2}=0,469 ; \mathbf{R}^{2}$ ajustado $=0,461$.

para explicar a variância do grau de motivação para prosseguir os estudos, como variável de desfecho, controlando-se o efeito de todas as outras medidas antecedentes. O modelo de regressão múltipla (stepwise) identificou quatro fatores preditivos, em ordem decrescente de contribuição: desmotivação, autoconfiança como aprendiz, escore do inventário de valorização do curso e motivação intrínseca para vivenciar estímulos. O coeficiente $R$ de correlação múltipla foi $0,69\left(R^{2}\right.$ ajustado $=0,46$ ). Outros componentes da EMA, rendimento acadêmico no primeiro ano, gênero e idade não foram fatores preditivos independentes.

\section{Discussão}

A versão do instrumento utilizada no estudo apresentou características semelhantes ao original no que concerne à consistência interna, ainda que os valores sejam um pouco inferiores. A estabilidade temporal das subescalas mostrou-se apenas moderada, em médio prazo, provavelmente refletindo as diferenças nas percepções dos estudantes de medicina sobre fatores motivadores em contextos distintos do curso. Essa interpretação é concordante com as diferenças significantes nas pontuações - elevação de identificação e queda de desmotivação - com a passagem do estudante da fase pré-clínica para a fase clínica do curso. É coerente, também, com a noção corrente de que componentes de motivação constituem estados dinâmicos e não traços estáveis de personalidade (Murphy \& Alexander, 2000; Zimmerman, 2000).

O perfil de respostas dos estudantes de medicina revela níveis elevados dos três componentes de motivação intrínseca e de motivação extrínseca por identificação, os quais são superiores aos níveis dos universitários estudados por Vallerand e cols. (1992), enquanto as diferenças de respostas por gênero foram menores. As discrepâncias entre os perfis podem ser atribuíveis a diversos fatores - de ordem cultural, social e acadêmica - presentes nas populações alvos dos estudos.

O sentido e a progressão das correlações obtidas entre as sete subescalas da EMA e as variáveis antecedentes e de desfecho estão alinhados com o continuum inferido da teoria de autodeterminação. O padrão postulado foi mais nítido nas correlações entre os componentes de motivação e o escore do inventário de valorização do curso, mostrando que as percepções de valor e significado nos desfechos de aprendizado estão associadas ao nível de motivação autodeterminada. $\mathrm{O}$ padrão equivalente observado nas correlações entre compo- nentes da EMA e o índice de motivação para prosseguir nos estudos sugere que a motivação autodeterminada tem bem mais força que o controle externo na intenção desses estudantes de persistir nas atividades da formação profissional.

Nessa perspectiva, os achados da análise de regressão são de especial interesse. Observou-se que fatores distintos, mas relacionados explicam boa parte da variância no grau de motivação para prosseguir os estudos. Estes fatores abrangem tanto característica mais individual, em termos de eficácia pessoal (autoconfiança como aprendiz) quanto característica de interação contextual (valorização do curso). No que tange à orientação, motivação intrínseca para vivenciar estímulos e (ausência) de desmotivação contribuem de forma independente para o desfecho em perspectiva. Por outro lado, o rendimento acadêmico (na forma de sumário de notas em disciplinas) não mostrou efeito independente, indicando que percepções subjetivas da vivência no curso são bem mais importantes do que o rendimento 'objetivo' para as expectativas dos aprendizes.

Em conjunto, os resultados reforçam a validade interna e externa do instrumento, embora suas propriedades pareçam menos robustas do que o indicado por Vallerand e cols. (1993). Essa atenuação pode ser atribuível a fatores diversos: perda eventual na versão, homogeneidade relativa do grupo, domínio específico e contexto realista das respostas.

Sob outra perspectiva, o estudo revelou um espectro de motivação principalmente autônoma entre os estudantes de medicina. A passagem da fase pré-clínica para a fase clínica não afetou a intensidade, mas sim a composição da motivação, sugestiva de ampliação de motivação mais autônoma. Entretanto, uma análise classificatória preliminar (dados não referidos) indica que um percentual de $25 \%$ dos estudantes tinham um espectro de motivação potencialmente problemático, ao término do primeiro ano de estudos.

Os achados têm implicações para o aconselhamento psicológico dos alunos e para a administração didático-pedagógica do curso. As discrepâncias no espectro de motivação dos estudantes sugerem a conveniência de acompanhamento mais estreito dos aprendizes e do desenvolvimento curricular.

Os resultados do estudo, em conclusão, dão suporte para a confiabilidade e validade de construto do instrumento, bem como para seu uso no estudo de motivação no ambiente universitário. Em particular, as indicações sobre o espectro de motivação dos estudantes de medicina e características correlatas apontam para a influência de fatores individuais e contextuais e para o caráter dinâmico desse espectro. 


\section{Referências}

Andrew, F.M., Klem, L., Davidson, T.N., O’ Malley, P.M. \& Rodgers, W.L. (1981). A guide for selecting statistical techniques for analyzing social science data. Ann Arbor: Institute for Social Science Research, University of Michigan.

Bandura, A. (1989). Human agency in social cognitive theory. American Psychologist 44, 1175-1184.

Deci, E.L. \& Ryan, R.M. (1985). Intrinsic motivation and self-determination in human behavior. New York: Plenum.

Deci, E.L. Vallerand, R.J., Pelletier, L.G. \& Ryan, R.M. (1991). Motivation in education: the self-determination perspective. The Educational Psychologist 26, 325-346.

Deci, E.L., Ryan, R.M. \& Williams, G.C. (1996). Need satisfaction and the self-regulation of learning. Learning and Individual Differences 8, 165-183.

Murphy, P.K. \& Alexander, P.A. (2000). A motivated exploration of motivation terminology. Contemporary Educational Psychology 25, 3-53.

Nehari, M. \& Bender, H. (1978). Meaningfulness of a course experience: a measure for educational outcomes in higher education. Higher Education 7, 1-11.

Norman, G.R. \& Streiner, D.I. (1994). Biostatistics: The bare essentials. St. Louis: Mosby.

Pintrich, P.R. (1991). Editor's comments. Educational Psychologist 26, 199-205.

Ryan, R.M. \& Deci, E.L. (2000). Intrinsic and extrinsic motivations: classic definitions and new directions. Contemporary Educational Psychology 25, 54-67.
Sobral, D.T. (1977). Alvos de carreira de alunos de medicina em Brasília. Revista Brasileira de Pesquisas Médicas e Biológicas 10, 265-270.

Sobral, D.T. (1992). Self-report visual scale of course appeal. Higher Education 23, 321-329.

Sobral, D.T. (1993). Motivação para aprender e resultados da aprendizagem baseada em problemas. Psicologia: Teoria e Pesquisa 9, 555-562.

Streiner, D.I. \& Norman, G.R. (1995). Health measurement scales. A practical guide to their development and use. Oxford: Oxford University Press.

Vallerand, R.J., Pelletier, L.G., Blais, M.R., Brière, N.M., Senécal, C. \& Vallières, E.F. (1992). The Academic Motivation Scale: a measure of intrinsic, extrinsic, and amotivation in education. Educational and Psychological Measurement 52, 1003-1017.

Vallerand, R.J., Pelletier, L.G., Blais, M.R., Brière, N.M., Senécal, C. \& Vallières, E.F. (1993). On the assessment of intrinsic, extrinsic and amotivation in education: evidence on the concurrent and construct validity of the Academic Motivation Scale. Educational and Psychological Measurement 53, 160-172.

Williams, G.C., Saizow, R.B. \& Ryan, R.M. (1999). The importance of self-determination theory for medical education. Academic Medicine 74, 992-308.

Zimmerman, B.J. (2000). Self-efficacy: an essential motive to learn. Contemporary Educational Psychology 25, 82-91. 


\section{Anexo}

\section{Por que venho à universidade?}

Usando a escala ${ }^{1}$ abaixo, indique - por favor - em que extensão cada um dos itens corresponde, atualmente, a uma das razões porque você vem à Universidade.

\section{Nenhuma correspondência}

1. Porque preciso do diploma, ao menos, a fim de conseguir uma ocupação bem remunerada, no futuro

2. Porque sinto satisfação e prazer enquanto aprendo coisas novas

3. Porque acho que a formação universitária ajuda a me preparar melhor para a carreira que escolhi

4. Porque gosto muito de vir à universidade

5. Honestamente, não sei; acho que estou perdendo meu tempo na universidade

6. Pelo prazer que sinto quando supero a mim mesmo nos estudos

7. Para provar a mim mesmo que sou capaz de completar o curso

8. A fim de obter um emprego de prestígio, no futuro

9. Pelo prazer que sinto quando descubro coisas novas que nunca tinha visto ou conhecido antes

10. Porque o curso me capacitará, no final, a entrar no mercado de trabalho de uma área que eu gosto

11. Porque, para mim, a universidade é um prazer

12. Já tive boas razões para isso; agora, entretanto, eu me pergunto se devo continuar

13. Pelo prazer que sinto quando supero a mim mesmo em alguma de minhas realizações pessoais

14. Por causa do fato que me sinto importante quando sou bem sucedido na universidade

15. Porque quero levar uma boa vida no futuro

16. Pelo prazer que tenho em ampliar meu conhecimento sobre assuntos que me atraem

17. Porque isso me ajudará a escolher melhor minha orientação profissional

18. Pelo prazer que tenho quando me envolvo em debates com professores interessantes

19. Não atino (percebo) porque venho à universidade e, francamente, não me preocupo com isso

20. Pela satisfação que sinto quando estou no processo de realização de atividades acadêmicas difíceis

21. Para mostrar a mim mesmo que sou uma pessoa inteligente

22. A fim de ter uma boa remuneração no futuro

23. Porque meus estudos permitem que continue a aprender sobre muitas coisas que me interessam

24. Porque eu creio que a formação universitária aumentará minha competência como profissional

25. Pela euforia que sinto quando leio sobre vários assuntos interessantes

26. Não sei; não entendo o que estou fazendo na universidade

27. Porque a universidade me permite sentir uma satisfação pessoal na minha busca por excelência na formação

28. Porque quero mostrar a mim mesmo que posso ter sucesso nos meus estudos

\section{Muita correspondência}

6

Total correspondência 6

1234567

1234567

1234567

1234567

1234567

1234567

1234567

1234567

1234567

1234567

1234567

1234567

1234567

1234567

1234567

1234567

1234567

1234567

1234567

1234567

1234567

1234567

1234567

1234567

1234567

1234567

1234567

1234567

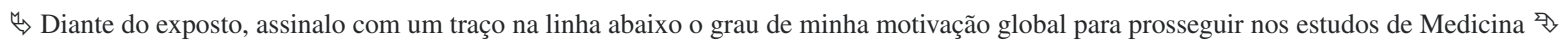
0 100

1 R.J. Vallerand e cols. (1992). Adaptação em 02/98 (DTS). Universidade de Brasília - Medicina. 